\title{
Effects of cooperation between a small and medium enterprise and AIST
}

\author{
— Impacts of the idea of "Monozukuri" on technicians -
}

\author{
Takafumi KomATSU ${ }^{1}$ and Shizuka NAKANO ${ }^{2 *}$
}

[Translation from Synthesiology, Vol.8, No.4, p.178-189 (2015)]

\begin{abstract}
Case studies and problem-solving steps are more important than new-technology transfer when collaborative studies are conducted between a small and medium enterprise and AIST. Detecting causes and issues takes considerable time, but, experiences obtained during collaborative research can decrease barriers and speedup progress. This report presents a case study on a collaborative project between Komatsuseiki Kosakusho Co., Ltd. and AIST to find a way to increase the life span of micro piercing tools. The solution, indirectly obtained by overcoming another problem is cost effective and easily applicable to factories, though it was not the surface coating technique considered at the beginning of the study. Through such experiences, it is possible to continue collaborative R\&D on this as well as other projects, e.g., within the "Operation Projects to Support the advancement of Strategic Core Technologies" framework of the Ministry of Economy, Trade and Industry.
\end{abstract}

Keywords : Cooperation, small/medium enterprises, problem-solving, manufacturing technology, joint research

\section{Introduction}

The major issue in conducting R\&D for manufacturing technology at AIST is that AIST does not have a site of production. No matter what advanced technologies are developed, since there is no environment to implement and evaluate such technologies directly, they often end up as an "armchair theory." Joint research with companies that engage in manufacturing is essential to prevent such theorizing and to execute practical R\&D. Since there are many excellent companies still in Japan, it seems that a joint research can be initiated smoothly and the results obtained swiftly, but the actual situation is not so simple. Fujimoto states, "At many small to medium companies in the manufacturing industry in Japan, the three factors, 'people,' 'things,' and 'money,' have been so finely appropriated that the companies must engage in competition with very few options, ${ }^{,[1]}$ and the resources of the companies have been depleted. In fact, in the age of corporate globalization process since the 1960s that includes (1) finding a footing in the local market, (2) exporting products abroad, (3) localized production, and (4) progression to multipolar production, ${ }^{[2]}$ it was important for the regional small/medium enterprises to establish relationships with major core companies of that region to ensure their management stability. To sustain business in such an environment, the priority of the managers of most small/medium enterprises was to fulfill the demands of the large companies, and to respond appropriately to the large companies' requests. The characteristic of small/medium enterprises was to focus efforts on specializing in certain fields as much as possible and to solidify their position within the hierarchy, and this was the most important concern of the managers.

However, the competition shifted to seeking the lowest possible cost of labor as a means to survive the cost competition. In the late 1980 s, the IT industry that saw growth in the domestic market and enjoyed competitiveness faced the cost reduction race due to market saturation and low-price sales at mass retailers, and many companies opted to transfer their plants overseas. The barrier of borders became lower among the corporate managers. Moreover, small/medium enterprises themselves were forced to take the global market into consideration.

Due to communication revolution such as the Internet, it has become possible to send large amount of data such as drawings to remote areas. The efficiency of distribution stimulated the movement of people, and an environment developed where shopping could be done easily on the Internet. A new market was born. Now there is a method to publicize and advertise one's skills, and direct business can be conducted with foreign customers by communicating in English that is not the mother tongue of either the customer or seller.

Recently, with the outflow of skilled workers through corporate transfer from advanced nations to emerging

1. Komatsuseiki Kosakusho Co., Ltd. 942-2 Shigakuwahara, Suwa, Nagano 392-0012, Japan, 2. Advanced Manufacturing Research Institute, AIST Tsukuba East, 1-2-1 Namiki, Tsukuba 305-8564, Japan *E-mail: shizuka.nakano@aist.go.jp

Original manuscript received October 15, 2014, Revisions received April 16, 2015, Accepted May 7, 2015 
nations, and through the introduction of state-of-the-art facilities due to the decreased opportunity to hand down skills at domestic sites of production, the difference in technology between advanced and emerging nations is rapidly decreasing. Through increased communication speed of vast information, enhanced education in the emerging nations, and increased number of students coming to study in advanced nations, there is a possibility that the advanced nations will eventually be surpassed by the emerging nations.

Some of the regional small/medium businesses in Japan are starting to advance globally utilizing technologies that may be the foundation of other fields, with highly specialized skills and experiences that were gained at the site of production. To succeed in international competition and for the domestic manufacturing to survive, it is necessary to push up the technological advantage that is vanishing to the forefront and to regain the manufacturing technology that overwhelms others. Collaboration with research institutions is a way to adapt to the new environment, but collaboration is extremely difficult in reality.

In this paper, we report a case study of joint research between AIST and Komatsuseiki Kosakusho Co., Ltd. (hereinafter, will be called Komatsuseiki), a medium-scale company in Nagano Prefecture. The report includes the issues encountered at the site of production, how progress was made in linking the R\&D without a site of production, what results were born including the changes in the "monozukuri (manufacturing)" mind, and what were the goals. Consideration is made on the effective ways of conducting joint research between research institutions and small/medium enterprises in the future. This paper is a merged work of Takafumi Komatsu, Executive Managing Director of Komatsuseiki and Shizuka Nakano, Advanced Manufacturing Research Institute, AIST. Initially, the plan was to divide the chapters and the authors write them individually, but we decided to merge the writings because each had different points for each topic. This chapter is also a combination of the manuscripts of the two authors. Therefore, please note that there may be places that may be difficult to understand.

\section{History of Komatsuseiki (Taking independent steps from below a large tree)}

Komatsuseiki was established in 1953 as an associate company for assembling wrist-watch parts for Daini Seikosha Co., Ltd. (currently, Seiko Epson Corporation). In its early days, it engaged in the assembly of watches as intended, and afterwards, retroacted upstream from manufacturing to production of dies to steadily widen its technological range.

However, as the watch market became saturated in the latter half of 1970s and sales expansion could no longer be expected, Daini Seikosha recommended the company to become self-reliant on businesses other than wristwatch parts. Fortunately, the company had fundamental technologies including pressworking, die polishing, cutting, and electric discharge machining for watch parts, and created a sales department necessary to apply such technologies to other fields. By responding to newly presented orders, the company was able to advance its pressworking technology for watches into the IT market.

The parts shown in Fig. 1, are the compact disk and hard disk suspension parts during the age when ten-megabyte hard disks were common. In addition to pressworking, a compound process was developed with laser welding that was state-of-the-art at the time done within the company to expand the technological range. However, the manufacturing time for dies could not catch up to the developing speed according to Moore's law, and with the overseas development of IT companies and the collapse of IT bubble in 2000, the IT parts business at Komatsuseiki declined rapidly.

Fortuitously, the manufacturing of automobile parts had started in the latter half of the 1980s due to the quality control technology that was nurtured in wrist-watchmaking. Since this involved supplying parts to products that directly affected human safety, the necessary quality assurance system was developed, as it was judged to be long-term, stable business. Also, since the issues of automobile parts were increased safety and environmental regulations, highprecision demands increased and the background was set for the utilization of wrist-watch technology.

The production scale of the orifices shown in Fig. 2, used as the electronic fuel injection parts for gasoline vehicles,

\section{Suspension for HD (during pressworking)}

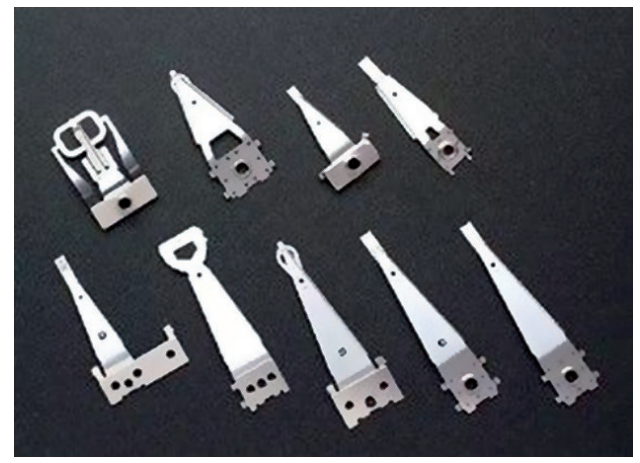

Suspension for $C D$

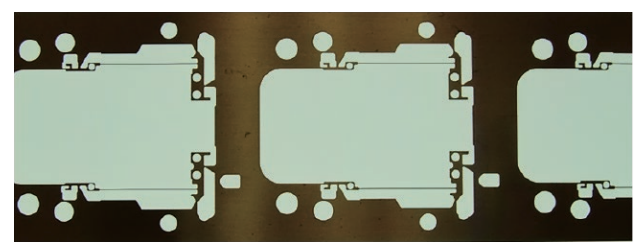

Fig. 1 Parts that were fabricated by Komatsuseiki by pressworking 
increased steadily from the late $1980 \mathrm{~s}$, and increased to monthly production of 3 million units in 2000 , and to monthly production of 5 million units in $2010 \cdot{ }^{[3]}$ In the course of such market expansion, with the strengthened environmental regulations that started from the Kyoto Protocol in December 11, 1997, the demand for small and high impact parts that were key to environmental measures intensified, and the advancement in technology and highefficiency production were expected to be sought in the future.

\section{Beginning of the collaboration}

The beginning of the collaboration between Komatsuseiki and AIST was at the nano tech 2008 exhibition (International Nanotechnology Exhibition and Conference). An on-demand processing system ${ }^{[4]}$ that was fabricated as one form of minimal fabrication technology was exhibited at the AIST booth (Fig. 3), and interest was raised there. The on-demand processing system was a small, automatic production facility that combined pressworking, aerosol deposition, ${ }^{[5]}$ heat treatment, and others, and aimed for flexible production and manufacturing according to the product to be made. In the exhibition, the processing of a MEMS scanner was being demonstrated. For pressworking, there was also a poster exhibition on increasing the lifespan of the micro-piercing punch. ${ }^{[6] 7]}$ Extension of lifespan for the micro-piercing punch was an attempt to increase the lifespan 16 times by treating the surface with a unique technology of gold ion implantation. Komatsu, who was the Section Chief of Production Management at Komatsuseiki at the time, was expecting increased order of fuel injection nozzles, and at the same time, faced the issues of reducing the die maintenance time and
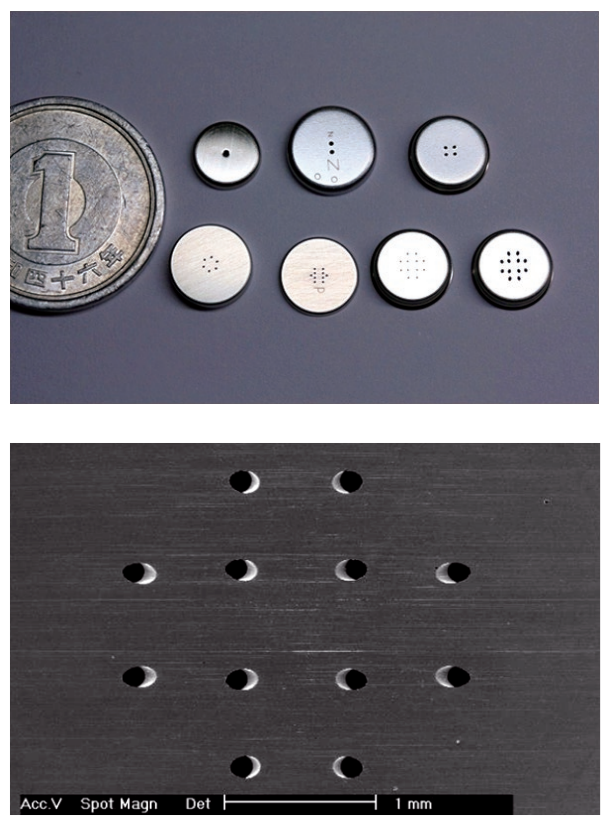

Fig. 2 Orifice plate for the gasoline fuel injection (top) and details of the orifice plate (bottom) increasing the surface area productivity to respond to the cost reduction demand.

From the side of Komatsuseiki, the explanation received at the exhibition seemed to provide a solution to the on-going issues. From the casual talks at the venue, a plan was born for a micro-piercing experiment, with the company providing a punch that was being created at the company, and gold ion implantation being done to the punch. From the decision that some direction could be gained from such "taste tests," this became the basis of the joint R\&D.

\section{Collaborative research and changes}

\subsection{Gold ion implantation punch experiment}

We shall present a brief explanation on the punch lifespan extension by gold ion implantation. As an issue of the piercing punch, the process involves punching and piercing of the material and retraction of the tool. Therefore, the punch reaches its maximum compression load at the time of piercing, is immediately relieved of stress, and reverses after reaching 0 speed. At that moment, it will be subject to tensile stress due to the relationship with the material. Due to the halting of movement and load in the reverse direction, there are issues of frictional wear and adhesion to the contact surface. Moreover, since the stress on the tool is inversely proportional to the processing size, the stress is equivalent to the strength of tool material for micro-piercing. While coating is mainly done to reduce this load, AIST developed a surface modification technology using the ion implantation method. The ion implantation method is a technology for adding ions and elements inside the material, and it is able to change the surface condition as a continuous structure with no boundary layer. While the film forming technology for coating has the issues of exfoliation or size change, ion

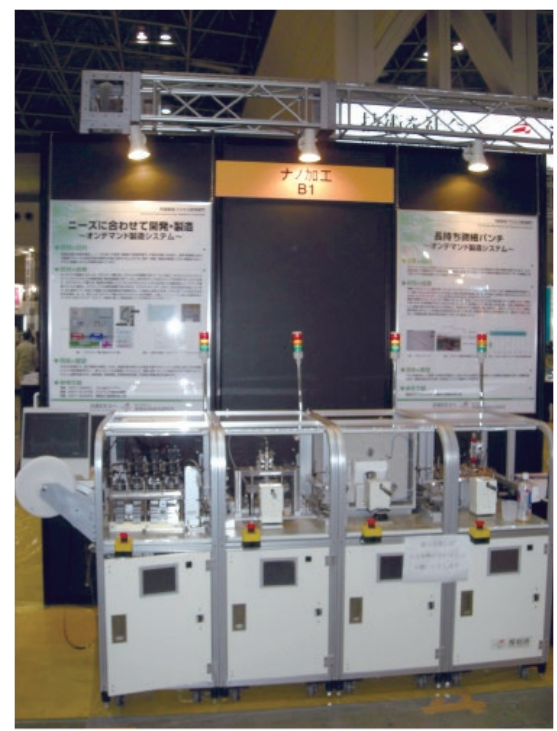

Fig. 3 Exhibition at the nano tech 2008 AIST booth in the Big Site venue. 
implantation removes such issues.

To extend the lifespan of the die tools, we worked on the solution that it is effective to achieve "a condition that is stable throughout the repeated process in pressworking." Therefore, we sought possibilities to obtain continuity of the carbide sintering structure, to allow softening to spread the stress, and to reduce adhesion and wear. Gold has large mass number and can take larger collision effect during irradiation than tungsten that is a carbide material. As a result, a small amount of gold can destroy the crystals in large amounts, and it is capable of changing the carbide surface into a nearly homogenous amorphous condition at small irradiance of 1 x $10^{16}$ atoms $/ \mathrm{cm}^{2}$ and low energy of $75 \mathrm{keV}$. By conducting annealing treatment after implantation, the amorphous carbide surface changes into tungsten trioxide. Here gold ion acts as an oxidation catalyst and oxidization occurs to a deep level in a short time. The film of tungsten trioxide, which contains cobalt tungsten compounds and cobalt oxides in the binder material, has small Young's modulus, and the coefficient of friction remains stable for a long period. Moreover, there is the effect of reducing surface adhesion, and the results lead to the extended lifespan of the punch. However, to introduce this method to the site of production, it is necessary to evaluate whether it can withstand mass treatment in an actual processing condition. Moreover, there were issues of cost reduction and whether actual manufacturing was possible, and the evaluation tests were conducted through sample provision.

In the sample provision scheme, the product manufactured at Komatsuseiki could be used directly, and the gold ion implantation was done at AIST immediately. Since internal evaluation of the processing test using the punch at the site of production was possible, which enabled verification in a relatively short time, the collaborative tests were approved. As a result, however, the goal for extending the punch lifespan was not achieved in the orifice processing test for the gold ion implanted punch. As shown in Fig. 4, it is thought that the method did not improve the punch lifespan because piercing was done at a 30 degree angle using a punch of $\varphi 0.2$

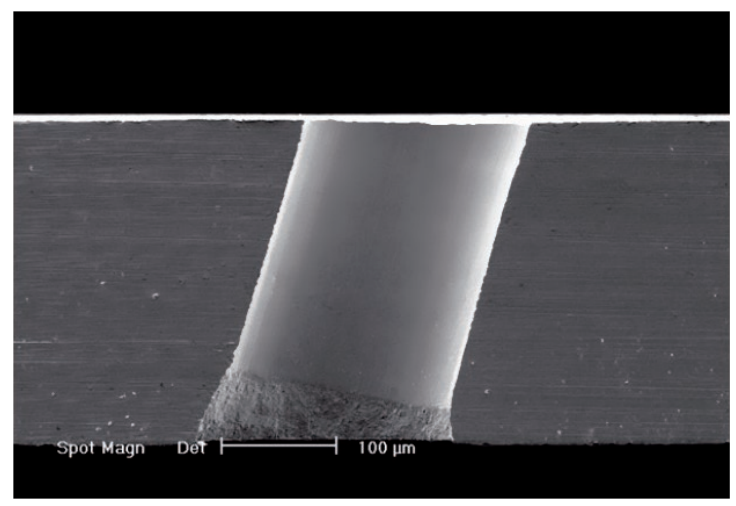

Fig. 4 Photograph of cross-section of angled piercing $\mathrm{mm}$, whereby the punch penetrated the material at an angle, the local stress occurred at the tip of punch, and the pressure spread out to the sides.

However, by conducting this joint research, Komatsuseiki realized the necessity to break down the phenomenon that was considered as one process of "angled micro-piercing" into smaller processes, and to understand the individual processes. Therefore, to understand the phenomenon inside the die that was difficult to evaluate, such as the analysis of the cause of damage including punch wear and the processing capacity of $\varphi 0.2 \mathrm{~mm}$ ultra-fine punch, the development of new evaluation methods was found to be necessary, and this led to joint research through a different perspective.

\subsection{Punch surface observation and development of micro-piercing force measurement}

While the visualization or bringing-into-sight of a phenomenon is the fastest method to find the cause of an issue and to seek the solutions, it is impossible to visually understand the phenomenon during die processing. In the past, studies have been done for experiments with glass, but sufficient evaluation could not be obtained since the conditions turned out different from the actual processing. Moreover, the evaluation at actual production volume would be even more difficult, and no study had been undertaken in the past. There was past research in which the die was disassembled regularly to remove the tool for evaluation, but in this method, the die had to be removed from the forming machine, disassembled, and then had to be reattached to the machine to continue the experiment. There was no reproducibility, it was difficult to conduct evaluation for every shot, and there was no case study for micro-piercing.

Therefore, AIST fabricated a die that enabled raising the punch (male part) to a visible position without taking the die apart, and fabricated an evaluation device that photographed the process with a microscope camera. ${ }^{[8]-[10]}$ Figure 5 shows the outline of the device. In FY 2009, the project received the "Monozukuri" Subsidy for Product Development Small/ Medium Businesses by the FY 2009 supplementary budget of the Ministry of Economy, Trade and Industry. Improvements

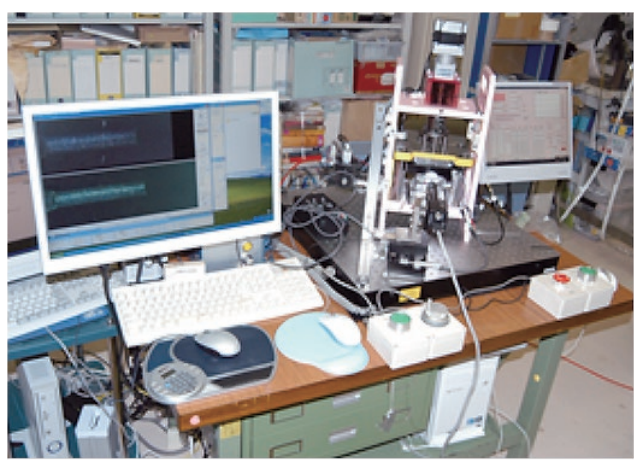

Fig. 5 Evaluation device for punch lifespan

Two cameras are used to observe the surface of the punch after every piercing shot. 
were made to enable tests at speed surpassing 40 SPM (shots per minute). It then became possible to photograph all shots for the test surpassing 5,000 10,000 shots that was close to the actual production level. Also, a load cell was attached to the punch and a laser displacement meter to the forming machine, to enable monitoring the change in load-displacement. During the experiment, we were able to observe the phenomenon where the carbide punch underwent plastic deformation and then broke. Using this evaluation device, we set out to understand the phenomenon in a complex process of angled piercing in which the processing phenomena were unknown, and to clarify the causes for punch fracture.

In the joint research, we fabricated the experimental die for angled piercing that matched the actual processing at Komatsuseiki, conducted the punching test for 5,000 20,000 shots, and observed the process of change leading to punch damage. Since extremely high precision was required for die adjustment, the Komatsuseiki engineers visited AIST to jointly conduct the experiment while exchanging opinions. An example will be shown from the results obtained. Figure 6 shows the results of punch observation in the 5,000 shot piercing test and the load-stroke curve. The image shows the adhesion of worked material and friction damage in the punch tip. We were able to directly observe when and where in the punch the adhesion or wear occurred. Figure 7 shows the same experiment under the same condition as in Fig. 6, but scrap clogging, which is one of the main die troubles, occurred. The buckling distortion of the punch occurred (a), the fracture occurred at the base of the punch in the next shot (b), and observations and the load-stroke curve of the phenomenon are shown. In Fig. 6, the scrap

\section{(a) Scrap clogging occurs (43rd shot)}
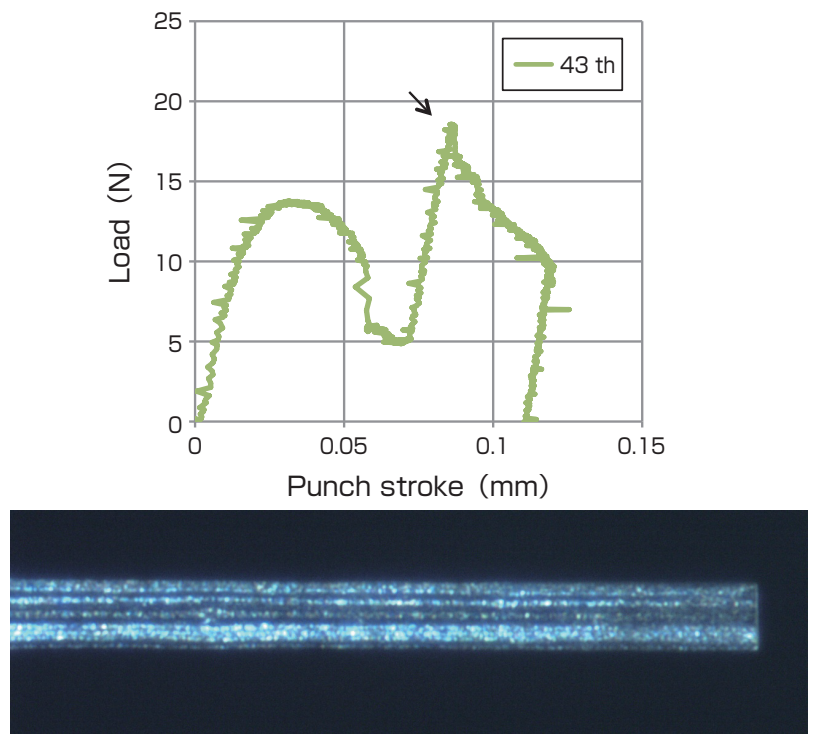

push load was even and was $5 \mathrm{~N}$ or less. However, when the trouble occurred in Fig. 7(a), the scrap push load increased to $18.6 \mathrm{~N}$. Although it is difficult to see in the image, slight warping occurred in the punch, and then it buckled. In Fig. 7(b), the scrap push load surpassed $20 \mathrm{~N}$, and the load was rapidly lost in two steps. Then the load decreased vertically. The punch broke at this point. While not shown here, the situation leading to the destruction of the punch was obtained as data, and the process and the moment of punch fracture were clarified along with the cause. From the results obtained in these tests, we created the method for detecting scrap
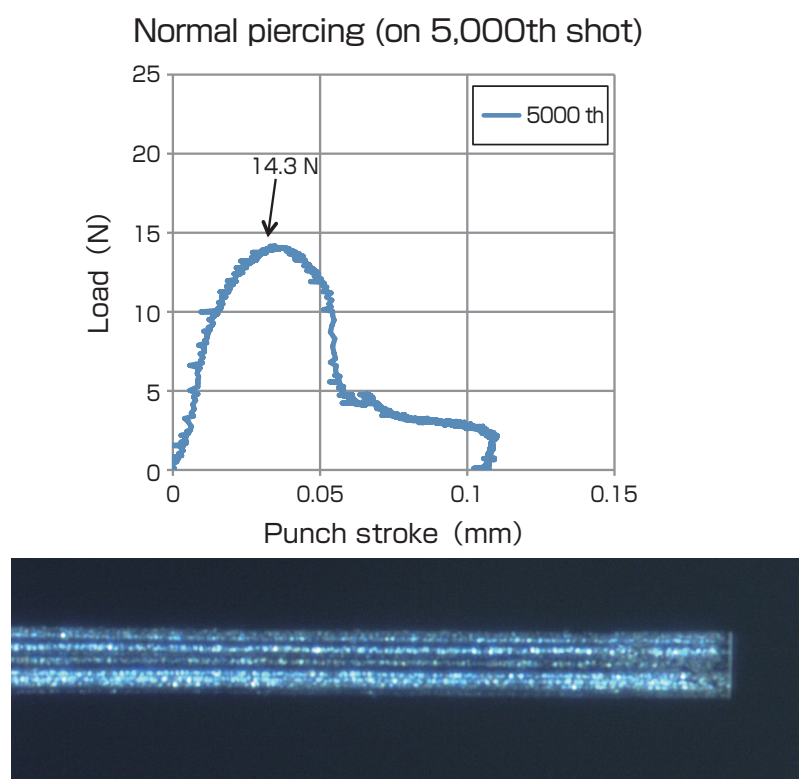

Fig. 6 Load-displacement curve during micro-piercing obtained by the evaluation device shown in Fig. 5, and the image of punch (the case when piercing has been done normally)

(b) Breakage due to punch buckling (44th shot)
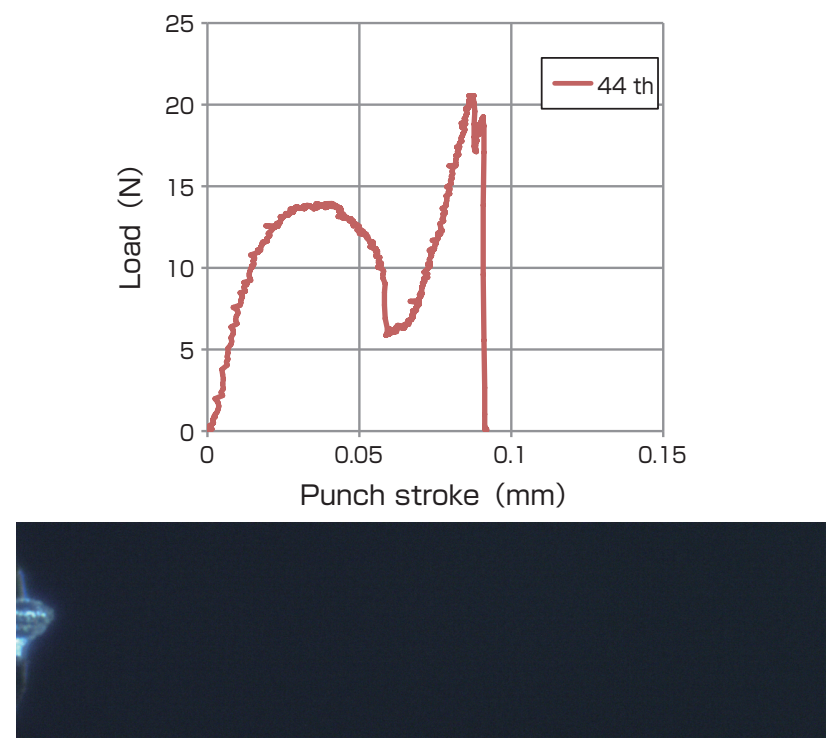

Fig. 7 (a) 43rd shot when the scrap pushing load (arrow) is high due to scrap clogging, and (b) 44th shot when breakage occurs at the base of punch due to buckling 
clogging and established countermeasures. ${ }^{[1]}$

The experiences of visualizing and understanding such a phenomenon were unprecedented even in companies that have specialized in pressworking, and we shifted to funded joint research as the expectation rose for problem solving through further research. As a result of repeated experiments, it was found that the maintenance level of the die was reflected in the changes in punch surface, and it became possible to evaluate the level of clearance adjustment in one shot. Although the clearance volume was only a few microns, an integrated evaluation tool was obtained considering the precision error of punch and die, slight eccentricity, positional precision with the die, imposition error, and others. Clearance is small in microprocessing, and the error relation with the precision of die making is very tight. In such a situation, we recognized that the effect of positioning during exchange of punch and die is very high. To be able to easily determine the condition of the die before processing continues contributes highly in reducing the burden of the maintenance worker. Also, as shown in Fig. 8, by preparing the material in midprocess and evaluating the distortion and hardness of the cross-section, we were able to clarify the state of materials

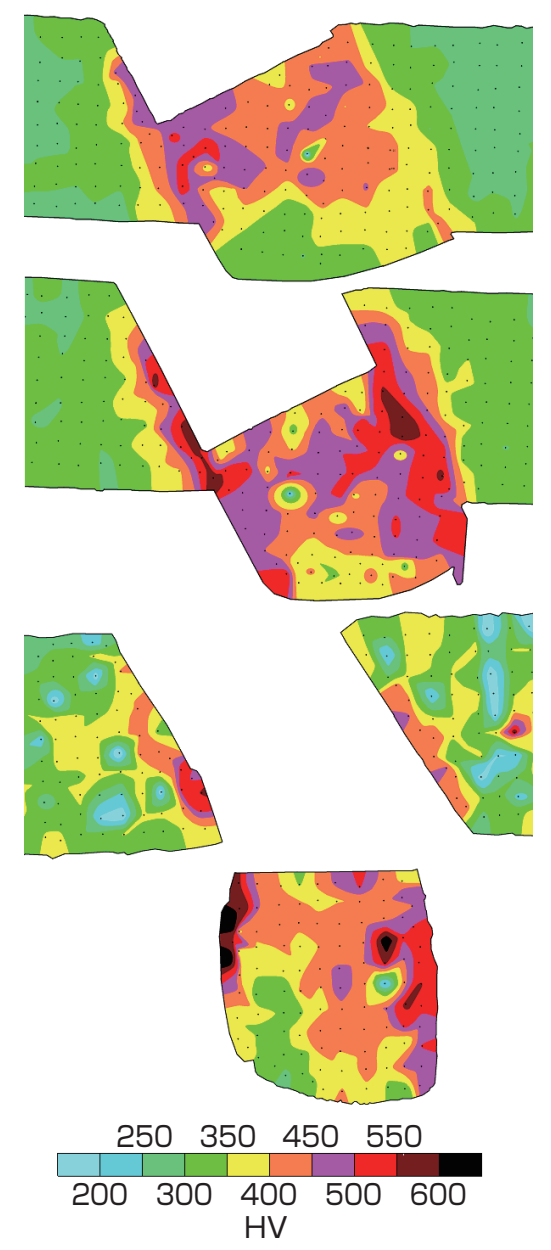

Fig. 8 Change in hardness distribution in the crosssection in angled punching ${ }^{[12]}$ that would become the product. ${ }^{[12]}$ Such visualization of processing enables breaking down the phenomenon of piercing that was thought to be a single process into four processes: (1) punch entry, (2) shearing, (3) pushing in the scraps, and (4) punch retraction. It then became possible to separately analyze the issues for each process.

Other diverse problems and phenomena are being elucidated, and it is desirable that solutions are generated that fill in the gap between research and actuality, such as cost consciousness, practicality, and attainment of reproducibility and stability. For example, anything that accompanies change in the procedure must be approved by the downstream user, and any addition to the existing procedure may be difficult depending on the content. Therefore, a method that can attain effectiveness without changing the procedure and can be accomplished by reviewing the current work method is likely to be employed. In this joint research, many findings were obtained for the initial issue, and the solutions including reconsideration of die size and thorough management involved hardly any additional cost, could be done in the company facility, and enabled repeated restoration to the same condition.

In manufacturing, the essential goal is to obtain a product and that it is desirable that it be of high quality, stable, and of low cost. On the other hand, research provides the means for a solution, but the means and the end are often confused. For example, even if the extended lifespan by surface treatment is superior and should be promoted, to be used in corporate production, equipment must be introduced, treatment condition must be optimized, and evaluation must be conducted during production. Only after such steps, the production can be started, but often the time required for such introduction is not available. It is necessary to discern the goal that the company demands and to provide diverse means. The difficulty of R\&D in manufacturing technology lies in this point, and while solutions cannot be obtained if one becomes involved in the method, research is not possible unless one becomes involved in the method. We believe it is effective to seek solutions while generating temporal gaps such as steadily going forward while taking diverse methods, extracting issues and problems in the company based on the accompanying analysis, and then taking the next step forward. While the results may be difficult to understand as tangible results may not be obtained or the result may not be clear at first glance, seen in the long term, it will be highly effective for the Japanese manufacturing industry.

This joint research progressed from the consideration of the processing method to the consideration of materials. Materials with fine metal crystal size were prepared, and the evaluation tests on how they would affect the process and product were started. Also, by analyzing the micro phenomena such as changes in the crystal structure during processing, the quality 
and processability of products were improved, which then developed into the manufacturing technology of advanced products. This led to the new phase, and the company strengthened its analysis environment by introducing the electron back-scatter diffraction (EBSD), and strengthened support of manufacturing by the construction of a system that allowed provision of test data to fulfill the customer's expectations. From FY 2013, industrial support themes were selected, and the development of varied shape micro-piercing, which demands a more advanced piercing process, and the development of metal micro-pumps that utilize the diffusion bonding technology ${ }^{[13]}$ were started. Here, based on the results obtained in the aforementioned joint study, we aim for highquality products by developing a die that incorporates a nanometer precision positioning stage that enables assembling precision and ease of maintenance. ${ }^{[10][14]-[16]}$

\section{Development in the corporate site of production}

Figure 9 shows the cause-and-effect diagram of pressworking for precision dies before the collaboration. The affecting factors are basically the $4 \mathrm{M}$, or man, material, method, and measurement, and each factor is followed individually in detail. The site of production was in charge of the part concerning people, the data provided by the material companies were used for materials, the die parts from the engineering division were incorporated, and the measurement device and production facilities selected by the production technology division were used to manage the quality control of the product.

These methods are set by the quality control standards such as ISO9001. No objections are likely to be raised as long as the company strictly follows and practices this ISO that has been translated into various languages. However, from a different viewpoint, since it is set by ISO, it is possible to be conducted by companies throughout the world, and it is not a factor that contributes to competitiveness.

By showing the flipbook animation of the punch surface and the change curve of the processing capacity during angled micro-piercing mentioned earlier, a certain reaction occurred at the site of production. By being able to visualize a phenomenon that could not been seen before or could not be explained before by words and images, the common expression of processing was now possible.

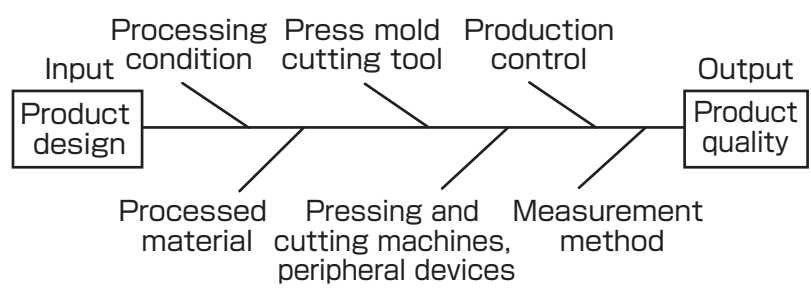

Fig. 9 Early cause-and-effect diagram
Through collaboration of joint research, a method different from trial-and-error that was done conventionally was developed, where the current situation was analyzed, the problem was defined, the solution was hypothesized, and the verification was done. When the test result was presented in the company, a certain change occurred not only among the researchers but also among the on-site workers. Until then, "angled micro-piercing" was considered to be a single process, but after the presentation, they started analyzing the process by separating it finely into (1) punch entry, (2) shearing, (3) pushing in the scraps, and (4) punch retraction.

Figure 10 shows the cause-and-effect diagram of the occurrence of burrs that are formed during angled microprocessing when processing orifices. This diagram was made and presented by the line manager. Compared to the earlier cause-and-effect diagram shown in Fig. 9, the problematic phenomena are refined and the depth of understanding and expression have changed dramatically. By refining the phenomena that were construed in only a few categories, the core cause is pursued and it has become possible to take specific actions for its solution.

The result greatly changed the management index called the "first run rate," that is the probability of a product becoming a completed product without becoming defective from the initial stage to the intermediate stage in a production lot. In 2011, the first run rate of Product A was less than $70 \%$. There were more than a dozen procedures, and $30 \%$ of the product that were produced in the first run became defective in a procedure somewhere along the way.

To overcome this situation, we looked at the activity steps including the on-site activities. We looked at the manufacturing process and management method of the product, difference in measurement by people, all the way to the movement of the punch during processing. For example, measurement of the hole position was done manually using tool microscopes, but because there were individual differences, we worked to reduce personal differences by shifting to image measurement.

Such action goals were led by the production site personnel under the slogan "100\% One Path Success." After four months of action, $90 \%$ was achieved stably, and $100 \%$ was achieved in five months. After that, over $95 \%$ is being achieved, and this site is positioned as a model line for training other workers and freshmen.

The results of joint research were shared through in-company presentations. In 2013, we succeeded in extending the punch lifespan by changing the size of part of the die and attaching the control standard. This was the initial goal when we started the collaboration for the research of the gold ion punch, but we reached the result by continuing the study 
using alternative methods and analysis, even though the initial method did not yield the desired effect.

\section{Changes in improvement thinking at the site of production through collaborative research}

It is difficult to set R\&D divisions in regional small/medium companies in terms of budget. A division that does not produce products is considered a division that does not earn money, and that brings about decrease of human, monetary, and material resources for research.

Komatsuseiki has 230 employees, and looking at the education level of the workers regardless of their ages, currently there are two people $(0.9 \%)$ working toward doctorates, five $(2.1 \%)$ with graduate school degrees, and $21(9.1 \%)$ who graduated from college. About $12 \%$ of the workers in the company have been involved in research. Of course, many have gained on-site experience after joining the company, and have engaged in problem-solving research, but most has resulted from customer demand, and it is difficult to conduct R\&D based on their own social or market projections. Even if there are researchers, there are only few manufacturing companies that actually engage in research, and how to fulfill customer demands is the priority for obtaining their daily bread. While the number of employees is increasing due to increased production scale and sales, the number of researchers are actually decreasing in companies that specialize in manufacturing.

To solve such a situation, it is important that the manager or the people close to management engage in collaborative research and share and develop the results in the company, in order for the small/medium manufacturers to expand to new areas. Particularly, when the scale of the company is small, the ability of the top managers tends to equal the ability of the company. Therefore, the overall level increase is accomplished efficiently in terms of cost through topdown rather than bottom-up, as the plans can be executed by short-term decisions. By creating success cases through small start-ups, more people can participate, the construction of collaborative body becomes easier, the consensus of company organization can be gained readily, and therefore, the scale can be increased gradually.

However, since the combinations of the talents of individual

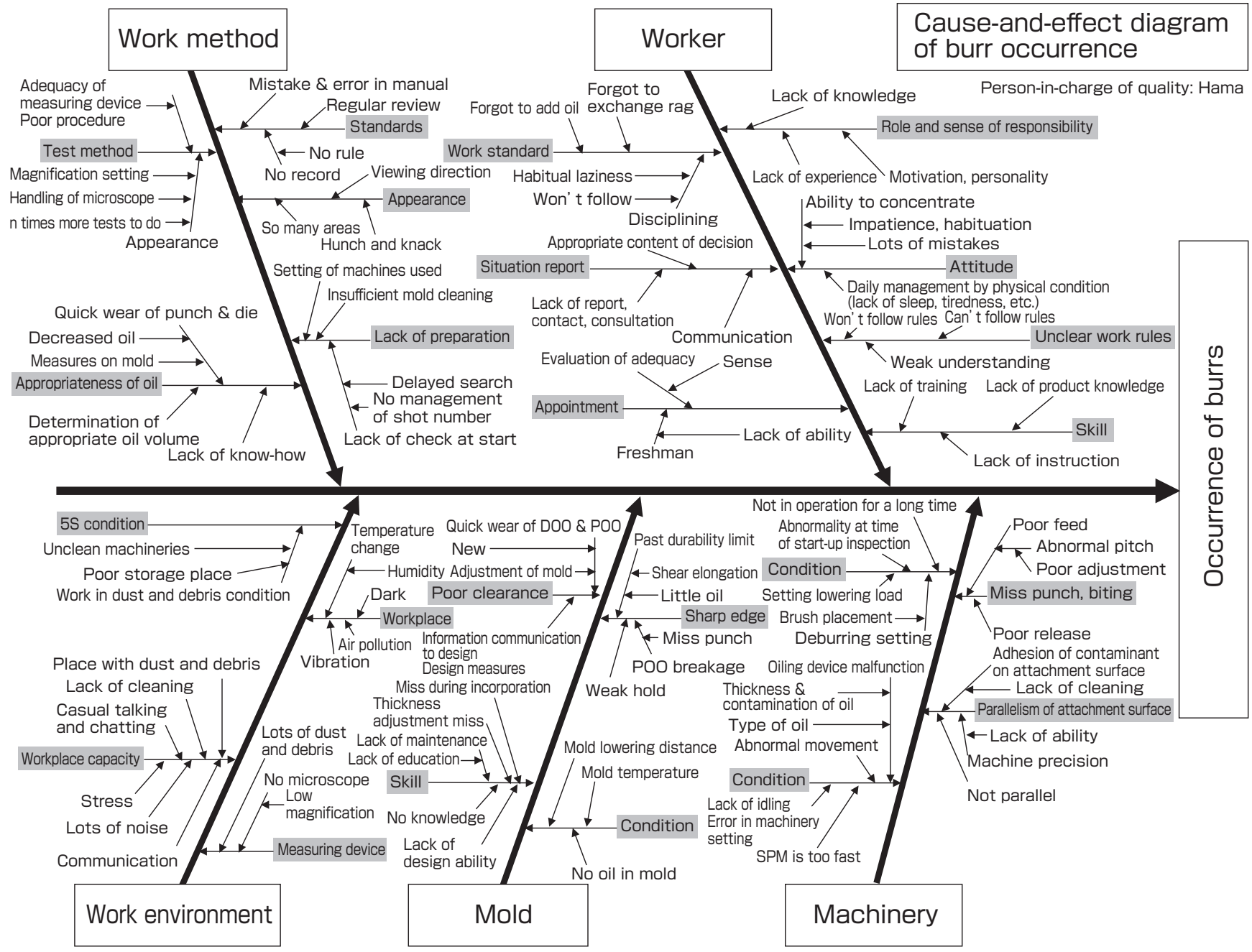

Fig. 10 Fig Detailed cause-and-effect diagram that focuses on the problem that occurred 
collaborative members who understand the market demand is infinite, the burden on the person-in-charge will be very high. In a small/medium company, only the top manager will be able to withstand such burden.

In joint research between small/medium businesses and research institutions, in many cases, the research is narrowed due to the focusing of the issue. This tendency is strong particularly in the joint research with universities, and there is a tendency to set one solution per one issue. However, in the research with AIST, solutions are obtained through multiple, comprehensive methods that are studied concurrently. In a small/medium company, since one person must cover much ground, research that runs close to the site of production is necessary as in this case.

At Komatsuseiki, the understanding deepened that new customers can be gained through the development of research results, and the collaborative research activities expanded the company's activity range including procurement of competitive budget, patent strategy, and participation in other projects through the relationship with the collaborative partner. In June 2013, a new R\&D office was opened, and the evolvement of research results is being done through the establishment of a new affiliated company. ${ }^{[17]}$

Seen from the side of AIST, there are plenty of research cases, but the application of results is difficult. Particularly in the development of manufacturing technology, it can be said that AIST does not have hands-on material because it does not have a site of production. Therefore, corporate joint research is important, but problems begin with terminology. For example, in research, "micron (micrometer)" is used regularly, while the company uses "x y-th" where the expression is in fractions based on millimeters. " $1 \mu \mathrm{m}$ " will be "one one-thousandth." Since the terminologies used at companies and research institutions are not necessarily the same, communication is difficult. In fact, as in the example of size explained above, the researcher construes the size "50 $\mu \mathrm{m}$ " as 50 times $1 \mu \mathrm{m}$, while the company considers it "onehundredth of $5 \mathrm{~mm}$ " or perceives it as half of one tenth of 1 $\mathrm{mm}$. Although the values are mathematically the same, the cognition is different. Since tolerance is utilized at the site of production, the "rough values" are also accepted as standard. In terms of awareness of numbers, for a researcher, $1 \mu \mathrm{m}$ against $50 \mu \mathrm{m}$ means that something is off by $1 \mu \mathrm{m}$ or there is a $2 \%$ difference, while on site, one one-thousandth against five one-hundredth seems to be a small difference. However, the production site has more experience struggling to process something at precision requirement of one one-thousandth scale. Nevertheless, in the joint research with Komatsuseiki, this gap in perception was significant. However, through monthly meetings to conduct joint experiments with the engineers, the communication level was mutually improved, and the researchers and engineers who could understand both perceptions were born. This is the greatest product of this joint research. In the technological discussions with Komatsuseiki, currently the discussion normally extends to the nanometer order, but people can switch freely from the expressions $100 \mathrm{~nm}$ or one one-ten-thousandth in their discussions now. In future research topics, new processing and manufacturing technologies are being developed. These will be works of near-boundary and boundary region surpassing the framework of current technology. In such cases, further communication ability will be required. From the age when one did not have to know Newtonian mechanics, one has entered the age when quantum mechanics is regularly used, and the agreement of perception, not only knowledge, is necessary. To accomplish this, it is necessary for people to engage in one experiment and be able to synchronize. Regardless of how excellent the results may be, transferring technology is not that simple.

\section{Conclusion}

The advanced processing technologies that were taken up by regional small/medium companies during the rapid economic growth period after World War II have become caught up in the globalization of companies that lead the game, the technological revolutions exemplified by the Internet, and the economic problems such as the Lehman shock. It is mandatory that they adapt to such environmental changes. The regional small/medium companies are exposed to global competition without exception. ${ }^{[18]}$ To maintain the competitive edge in such an environment, problem solving by collaboration rather by individuals is one method for competitiveness and differentiation. In companies that have little change in employee composition, there is a limit to the problem-solving abilities and it is difficult to shorten the distance between the customers.

To capture the diverse or niche demand of the customers, it is necessary to deepen understanding of the people with whom one might collaborate and their skills and knowledge, and to work together organically. This will enable building of competitiveness unseen before, and shall also create new customers.

Many regional small/medium companies are reluctant to promote collaborative research due to the limit of "people," "material," and "money." However, by building the research environment through small start-ups and then expanding and developing into the site of production, it is possible to obtain effectiveness in various new ranges.

From the side of research institutions such as AIST, collaboration with small/medium companies that engage in manufacturing is essential. On the other hand, research institutions tend to think that the results can be used as is, but many of them are indirectly related to manufacturing. 
At actual sites of production, manufacturing methods are different for each product actually made, the issues are of infinite variety, and the case where direct application is possible is rare. Also, companies are often seeking direct solutions. Indirect practices involve the search of causes, the pursuit of solutions, and the final attainment of solutions, but the case is often, "the situation is this right now, and we need a solution right now," without the search of the cause. Companies often see a phenomenon as simple saying, "it was running well until now" or "it doesn't go well for new products," and is often led to thinking that "there is no problem in the way we use to do it" or "the bad part is only this (the actual trouble) and all will be well if this is fixed." However, the reason "it was running well until now" is because the conditions were good or they were "lucky." Particularly for manufacturing technology that existed for a long time, the difficulty increases daily, and many companies may not be aware of this. In the most advanced fields, work is being done at levels that are not written in text books, and the way of thinking about the issue is different. When one does not realize that things are becoming difficult, the consciousness turns to "it must go well," and fails to seek the essence. In the joint research with Komatsuseiki, the experience where "all went well" worked against the situation and the cause of the real problem could not be seen. AIST researchers worked to find the cause of the problem by analyzing the reasons why things did not go well one by one, without relying on the newly made technology. As a result, the solution of the problem was obtained, and the company attained consciousness for "looking at more difficult issues" and "seeking solutions at a higher level" through the process, and built the strong will to engage in the search of the cause. In this joint research, at the beginning of the development, the question "isn't there a problem in this area?" was brushed off with "there was no problem there before." However, the cause was finally found by clarifying the individual factors of the issue. In retrospect, I think this detour was a necessary process by which the company and the research institution found common ground, and it can be said that the detour became the foundation for tackling the next issue and more difficult products. From the last fiscal year, we started tackling the joint industrial support theme, and we are engaging in difficult, high-level technological development that enables the manufacture of micro-precision products that could not be made with conventional technology. The current joint research is for the technology to visualize the invisible process in order to accurately analyze technology. This is like adding new pages to a textbook, but it will increase the knowledge and experience of the engineers and provide easier progression to difficult processing for the company. In the industrial support program, we aim not only for a single company, but mass production through collaboration with multiple companies including the upstream material manufacturers and downstream final product manufacturers, as well as research institutions such as AIST and universities.
Since the company has $30 \%$ of the world share, it is trying to win the competition against the world in the next generation, without being afraid of taking a detour. The company must accept taking the long way, and the research institution must be willing to take time to handle matters carefully. As a result, we can get new, difficult, and high added-value products. Although the advancement of manufacturing technology is the basis of creating new industry, time is necessary. It is necessary that a technology be effective in the next development of processing with stricter conditions than the one that is being worked on, and it is also necessary that the effectiveness be visible. Pressworking is a processing technology that has been used since ancient times. Some people think that it is "past technology" and "it is sufficiently handled by companies," and it is declining as a research theme for universities and research institutions. However, the actual corporate production sites are perpetually challenged with new issues. Solutions cannot be obtained simply by "buying new devices" or "buying new technology," and only when the cause is found and a true solution is obtained, one can rise higher. In this sense, the role of a research institution is important.

I hope this paper will give some guidance to the future joint research between research institutions and companies.

\section{References}

[1] T. Fujimoto: Monozukuri Keieigaku (Manufacturing Business Administration), Kobunsha (2007) (in Japanese).

[2] K. Ohmae: The Borderless World, Harper Collins Publishers (1990).

[3] T. Komatsu, M. Komatsu, H. Yanagisawa, T. Uehara, T. Yanagisawa and S. Manabe: Development of advanced stamping process of orifice plate for electric controlled fuel injector: Contribution of small parts to environment, Journal of the Japan Society for Technology of Plasticity, 52 (611), 1281-1285 (2011) (in Japanese).

[4] S. Nakano and K. Ashida: Minimal manufacturing of developing factory system for micro electro-mechanical systems devices, Journal of the Japan Society for Precision Engineering, 77 (3), 254-258 (2011) (in Japanese).

[5] J. Akedo, S. Nakano, J. Park, S. Baba and K. Ashida: The aerosol deposition method: For production of high performance micro device with low cost and low energy consumption, Synthesiology, 1 (2), 130-138 (2008) (in Japanese) [Synthesiology English edition, 1(2), 121-130 (2008)].

[6] S. Nakano, K. Ito, T. Inoue, M. Yoshida and H. Ogiso: Improved lifespan of micro-scale punch tools by ion implantation, Trans. Mat. Res. Soc. Jap., 32 (4), 865-868 (2007).

[7] S. Nakano, Y. Ming, M. Yoshida and H. Ogiso: Surface damage of gold-ion implanted Co-WC micro-punch tools during press processing, Trans. Mat. Res. Soc. Jap., 36 (1), 83-86 (2011).

[8] S. Nakano, T. Shiratori and Y. Suzuki: Development of visualization technology on fine shape press working: Case of fine round hall stamping, Sokeizai, 53 (7), 22-26 (2012) (in Japanese). 
[9] S. Nakano, T. Shiratori, Y. Suzuki and M. Katoh: Bisai nukiuchi kako no kashika jikken (Visualization test for blanking micro pores), Proceedings of the 62nd Japanese Joint Conference for the Technology of Plasticity, 441-442 (2011) (in Japanese).

[10] S. Nakano, Y. Suzuki, T. Aihara and T. Shiratori: Visualization technology and nanometric positioning die system for micropiercing, Journal of the Japan Society for Technology of Plasticity, 56 (650), 213-218 (2015) (in Japanese).

[11] T. Shiratori, S. Nakano and Y. Suzuki: Shokeiko nanameuchinuki kaju no kanshi ni yoru kasu-zumari kenchi (Scrap clogging detection by monitoring small-diameter angled piercing load), Proceedings of the 62nd Japanese Joint Conference for the Technology of Plasticity, 443-444 (2011) (in Japanese).

[12] M. Katoh, T. Shiratori, Y. Suzuki, S. Nakano and T. Komatsu: Bisairyu soshiki o yusuru SUS304 gokuusuita eno bisaiketsu no naname-nuki ni okeru zairyo henkei kyodo (Material distortion behavior during angled micro-piercing in ultra-thin SUS304 plate with micro-granular structure), Journal of the Japan Society for Technology of Plasticity, 55 (638), 223-227 (2014) (in Japanese).

[13] M. Katoh, T. Shiratori, N. Sato and Y. Suzuki: Austenite-kei stainless-ko no soshiki seigyo ni tomonau kakusan setsugo ni oyobosu tansoryo no eikyo (Effect of carbon content in diffusion bonding accompanying the structural control of austenite stainless steel), Proceedings of the 65th Japanese Joint Conference for the Technology of Plasticity, 99-100 (2014) (in Japanese).

[14] S. Nakano, Y. Suzuki, T. Aihara and T. Shiratori: Nano stage kumikomi kanagata ni yoru center clearance bisaiko-nuki kako hyoka (Evaluation of micro-piercing center clearance by nano stage built-in die), Proceedings of the 65th Japanese Joint Conference for the Technology of Plasticity, 215-216 (2014) (in Japanese).

[15] T. Shiratori, S. Nakano, Y. Suzuki and T. Aihara : Nano stage kumikomi kanagata ni yoru die no ichi chosei ga bisaiketsu kirikuchi-men ni ataeru eikyo (The effect on micro hole orifice due to position adjustment of die by nano stage built-in die), Proceedings of the 65th Japanese Joint Conference for the Technology of Plasticity, 217-218 (2014) (in Japanese).

[16] Y. Suzuki, T. Shiratori, S. Nakano, M. Katoh and T. Aihara: Austenite stainless ko no kako ondo ga bisaiketsu-nuki kako ni oyobosu eikyo (The effect of processing temperature of austenite stainless steel on micro-piercing), Proceedings of the 65th Japanese Joint Conference for the Technology of Plasticity, 219-220 (2014) (in Japanese).

[17] K.K. Nano Grains HP: http://www.nanograins.co.jp/, Accessed 2015-04-30 (in Japanese).

[18] T. Komatsu: Chiho chusho-kigyo no sangakukankin renkei no jirei to renkei no merit to demerit (Case study of collaboration among industry, academia, government, and financial institution for local small-medium businesses and advantages and disadvantages of such collaboration), Journal of the Japan Society for Technology of Plasticity, 54 (632), 787-791 (2013) (in Japanese).

\section{Authors}

\section{Takafumi KomATsu}

Graduated from the Department of Mechanical Engineering, Tokyo Denki University in 1995. Studied business administration in Ireland and England from 1995 to 1999. Joined Komatsuseiki Kosakusho Co., Ltd. in 1999. Person-incharge of Production Technology; Section Chief, Production Management; General Manager, Manufacturing; and currently, General Manager of R\&D and Executive Managing Director. Established nano grains Co., Ltd. in 2014 and became President. In this paper, wrote about capturing the specific issues at the site of production and promoting their solution, and the results of collaboration with AIST and the changes that occurred in the company due to such collaboration, from the perspective of person in charge of promoting joint research on the corporate side.

\section{Shizuka NAKANO}

Completed the master's program at the graduate school of University of Electro-Communications. Joined the Mechanical Engineering Laboratory in 1989. Dispatched to the New Energy and Industrial Technology Development Organization (NEDO) in 2001. Received Doctor of Engineering from University of Electro-Communications in 2003.

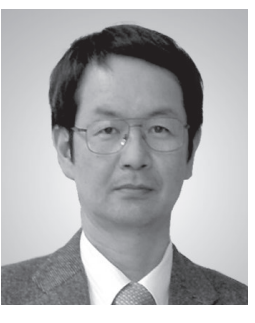
Worked on the development of manufacturing technology with emphasis on ion beam processing, Micro Machine Project. Other than the fine shape pressing presented in this paper, currently studies minimal fabrication and metal layer formation technology. Group Leader, Function Forming Group, Advanced Manufacturing Research Institute, AIST. In this paper, worked on the visualization of pressworking, the development of surface treatment punch, as well as carried out the visualization technology and its analysis/evaluation in the joint research with Komatsuseiki Kosakusho Co., Ltd.

\section{Discussions with Reviewers}

\section{Overall}

Comment (Naoki Ichikawa, Advanced Manufacturing Research Institute, AIST)

This paper describes an example of problem solving in micropiercing for pressworking, and the collaboration between AIST and a small/medium company is described through each other's perspectives. The importance and necessity for regional small/ medium companies to collaborate with AIST or universities due to changes in business circumstances are explained. Also, communication between the company and AIST, sharing of awareness of the problem, mutual trust and honest proposals, and suggestion of wide-ranging technologies that may lead to problem solving are described along with the progression of the research topics. It can be seen that the process is important in handing over the technology to small/medium companies.

It is specifically mentioned that there are differences in the ways that researchers and on-site engineers think about problem solving of manufacturing technology, as well as how such 
differences are surmounted to form the collaborative relationship to solve the issues. This paper is important as a success story of an approach to transfer the manufacturing research result to the site of production. The point of success in collaboration between researchers and production sites is that the way of thinking for problem solving by the on-site engineers changed as the pressworking process became visible.

\section{Initiation and background of the collaboration Question (Naoki Ichikawa)}

The authors describe that the evaluation method and visualization of the phenomenon inside the die as well as punching force measurements were developed in the process of conducting the gold ion implantation experiment, but I think the authors should provide a little more explanation from the company side. It is written that when the experiment was started, the company approved of the point that the evaluation and experiment could be done relatively easily with the existing method, and I believe the company's initial expectation was that the gold ion implantation would extend the lifespan of the punch. However, the experiment did not progress as planned and the gold ion implantation did not do well, yet it was decided the joint research would be continued due to the evaluation results obtained. I would like to know how the company looked at this result, and what was the point that made it decide on the continuation of the joint research.

\section{Answer (Shizuka Nakano)}

For the gold ion implantation punch, the situation differed between the laboratory and the production site, and the AIST side was aware from the beginning that direct introduction of technology would be difficult. Particularly, angled micropiercing was an extremely difficult process that could not be done normally, and it was a difficult research topic, and we were careful in exchanging dialogue sufficiently right from the moment we received the samples. We provided topics for exchange of dialogue, such as having people visit AIST to take a look at our research tools and results, including our main facility that was the evaluation device for the lifespan of die tools. Moreover, we discussed the issues that would be expected when these were brought to the site of production. Since the company plant itself was the aggregation of know-how, we were unable to visit at the time of sample provision in the beginning of research. It was rather difficult for the researchers since we had to entrust the tests to the company. Later we heard that the company had thoroughly tested all methods that it could think of, and was interested in us because we were "developing some weird technology that they had never heard of before." (In fact, ion implantation takes a lot of explanation to gain sufficient understanding.) The fact that the company was relying on the possibility for the "unknown" led to this collaboration.

Also, explanation of the importance of knowing the process and to grasp the situation in the experiment using the provided sample led to the awareness that "phenomena inside the die are really not well known." I think it was good that we landed there. As it is written in the text, I was reminded clearly from start to finish that communication is important.

\section{Answer (Takafumi Komatsu)}

For the company, we would obtain the short-term effect if the extension of the punch lifespan was realized by gold ion implantation, but besides that, the management was expecting the long-term effect of generating new ways of looking at things through the collaborative research with AIST. As a result, the extension of the punch lifespan by gold ion implantation was not achieved, but it was clear that new ways of looking at pressworking emerged in our company, and we decided to continue the joint research.

\section{Visualization of punch experiment Question (Naoki Ichikawa)}

Since it is difficult to see the die in action, I think the point here is to look at the punch by raising it to the position that it can be observed. From the company side, what was the impression when you saw the changes, and what future possibilities did you perceive from this?

\section{Answer (Shizuka Nakano)}

In pressworking, we have always wanted to look at what was going on, but it was something we couldn't see because: 1) if it is made of transparent material, we can only process materials that were weaker than the die, 2) if the process is divided, the condition changes, and 3) if the die is taken apart each time for evaluation, only evaluations for very small number of pressing steps can be done. We roughly understood the situation based on the long hours of research activities by our forefathers. However, due to the advancement in processing, more detailed analysis was necessary, and it became important to seek the essence of processing such as for micro-piercing and complex shapes for which solutions could not be found by past theories. Therefore, the importance certainly increases for technologies that enable seeing or estimating what cannot be seen. In the lifespan evaluation device, we were not looking at the moment of pressing, but were able to build a system for observing the tool surface for each piercing, and this was realized at the actual production level although it was a little slower at 40 SPM (shots per minute). It achieved the level that allowed a simulation test of an actual pressworking plant. Moreover, it is important to capture "the abnormalities that occur sometimes" and to review them by repeating similar experiments several times. The "phenomena that occur all the time" are issues that are easy to find solutions for, but "phenomena that occur sometimes" are difficult to grasp, and are actually the biggest problems at the site of production. Since they are probabilistic phenomena, it is considered that to run the process a number of times is the only way to get to the problem. However, we were able to create a technology to catch and hold this slight probability, and this is the point that matched the company's demand.

\section{Comment (Takafumi Komatsu)}

The company side was thinking that it was impossible to see the changes in tools after each punching process, but that assumption was overturned in this research. What we could not see became visible, and we expected new impact at the site of production. As a result, understanding deepened at the site of production as the phenomenon was broken down and the measures were taken according to the base cause. That matched the initial expectation.

\section{Question (Naoki Ichikawa)}

The authors state clearly that "there was no research or case study," but I think it would be better to consider using the expression "such work has not been done." In fact, I think there are studies that evaluate each shot, though not for micro-piercing. Answer (Shizuka Nakano)

For example, a method of evaluation after 1,000 shots where the die was disassembled had been done at the Mechanical Engineering Laboratory. This was punching for holes with a diameter of $10 \mathrm{~mm}$. The issue in this case was since the die was disassembled, the same condition could not be reproduced after the die was reassembled. Also, I have not found evaluation for shot-by-shot even for micro-piercing of $10 \mathrm{~mm}$ class. The reason is fairly clear, as certain conditions are needed for pressworking under strict conditions that is equivalent to the stress on tools for micro-piercing. One is the case where the plate thickness is thicker than the hole diameter. This is the case where an $8 \mathrm{~mm}$ diameter hole is punched into a $10 \mathrm{~mm}$ plate, but there are very 
few examples. The next condition is the processing precision of the tools, but in the current situation, the processing precision of the tools is not different for micro-piercing and normal size piercing. That is, micro-processing must be done at a condition with relatively low precision. The processing tolerance for the tool in the case where a $8 \mathrm{~mm}$ diameter hole is punched into a 10 $\mathrm{mm}$ plate can be realized at $1 \mu \mathrm{m}$. With tight clearance of $5 \%$, there is a gap of $0.5 \mathrm{~mm}(500 \mu \mathrm{m})$, and processing can be done by combining the tools with processing tolerance of $1 \mu \mathrm{m}$. In this case, it is clearance: tool precision $=500: 1$. On the other hand, the processing tolerance is about $1 \mu \mathrm{m}$ for micro-processing. Since the $5 \%$ clearance for plate thickness $0.1 \mathrm{~mm}$ is $5 \mu \mathrm{m}$, it is clearance: tool precision $=5: 1$. Since sufficient precision is not attained in the current micro-pressworking, the issues of lifespan and others show up clearly. On the contrary, for macro-processing, relatively high-precision tools are used, whereby the wear and adhesion become relatively small, and it was not necessary to thoroughly consider the lifespan issues. In experiments at ordinary scale, the effect of one shot is small, and there was no need to follow the change after every shot. In micro-processing, the effect of one shot is relatively large, and our research was necessary.

\section{Points for successful collaboration \\ Question (Naoki Ichikawa)}

Please organize the points for successful collaboration and the specific course of development. Please present clearly what goals and which actions were taken at each step of evaluations and reviews at the company side, and how the differences in thinking, such as the opinions of the partners, were overcome (or how compromises were made). You say that you obtained results that satisfied the company by visualizing the fine changes in conditions using the observation device. How did you fill in the gap between what the company was demanding?

Answer (Shizuka Nakano)

What was most effective, I think, is that we gradually came to an agreement through exchange of opinions done every month during the experiments at AIST. Since the experiment involved repetition and the experimental device was automated, we set and started the device, and we exchanged opinions while watching the image or the load data as the experiment ran. If there were abnormalities during the experiment, we considered the cause, and if there were no abnormalities, the experiment ran on, and we talked about the expected causes and other topics. In doing so, the thinking that seemed to be quite apart came closer together, and as a result, we reached the point of "how to evaluate the issues at the site of production." Although the details cannot be presented because it is the actual know-how, for the final on-site evaluation, I made a rather difficult request to the workers at the plant. Since the person-in-charge at Komatsuseiki could take responsibility and appreciated the value of carrying out the experiment, it was done, the final proof of cause was confirmed, and this led to the result. I think this was achieved by us going about "stubbornly," "never giving up," and by "not jumping to conclusions."

Comment (Takafumi Komatsu)

In joint research between small/medium businesses and research institutions, in many cases, the research is narrowed due to the focusing of the issue. This tendency is strong particularly in the joint research with universities, and there is a tendency to set one solution per one issue. However, in the research with AIST, solutions are obtained through multiple, comprehensive methods that are studied concurrently. In a small/medium company, since one person must cover much ground, research that runs close to the site of production is necessary as in this case.

Question (Seigo Kanemaru, Electronics and Manufacturing, AIST)

To understand the points of successful collaboration in this paper, please consider the following. In working on visualization, what kind of discussions did you have with the on-site engineers on what to make visible, and what kind of alterations did you make to the evaluation device? As a result, you mention that the pressworking cause-and-effect diagram was refined and this was effective in improving the production procedure. What were the reasons that enabled the refinement of the cause-and-effect diagram? If you have a specific example of such a diagram that was applied to on-site activities, I think it will further clarify the effect of visualization.

\section{Answer (Shizuka Nakano)}

For the on-site engineers, we started with the points, "what can be seen in this research" and "what is actually seen on site." In fact, it's "there are things that cannot be seen in research" and "people on site think they know it all," but we had people understand the meaning of each individual data, the meaning of change of load in the interim process, as well as the point that a process that seems to happen in only a few milliseconds is actually separable into multiple processes and that their situations change from moment to moment. The initial evaluation device was slow, and a camera was on one side only, but for the research with Komatsuseiki, cameras were installed on both sides and high speed was achieved. The production speed of pressworking is fast, and high speed was necessary to understanding the phenomena occurring there, and cameras on both sides were necessary to ensure there were no missed shots. Of course, there is progress between the first experiment and the latest one, and the complexity has increased considerably. There has been evolution where the level of processing that could not be achieved at the start of research is now being done normally at the production level, and this has become possible because we understood the phenomena well and the problems were captured accurately. 